(C) Facultad de Ciencias Biológicas UNMSM

\title{
Diversidad y el estado de conservación de cuerpos de agua Amazónicos en el nororiente del Perú
}

\section{Diversity and state of conservation of Amazonian waterbodies in the Northeast of Peru}

\author{
Hernán Ortega, Blanca Rengifo, Iris Samanez y Carlos Palma
}

Museo de Historia Natural, Facultad de Ciencias Biológicas, Universidad Nacional Mayor de San Marcos. Apartado 14-0434, Lima 14, Perú.

Email Hernán Ortega: hortega@terra.com.pe

\section{Resumen}

En el presente trabajo se describen la diversidad y estado de conservación, determinados por un inventario biológico rápido, entre el 24 y 30 de octubre de 2005, en cuerpos de agua del nororiente del Perú, ubicados, entre los 133 y 680 m de altitud, entre Tarapoto (San Martín) y Yurimaguas (Loreto). Se colectaron datos y muestras en 26 estaciones. Los peces fueron colectados con redes de arrastre a la orilla, el bentos con red "Surber", y el plancton con red estándar (40 micras). En cada estación, se anotaron coordenadas (UTM) y se describieron hábitats limnologicamente $(\mathrm{pH}$, temperatura, conductividad, oxigeno disuelto). La riqueza de especies en peces fue de 95, predominan Characiformes y Siluriformes. La riqueza de especies de peces en fitoplancton fue de 74, en zooplancton, 22 especies y en bentos de 20 especies. Para determinar el estado de conservación se uso el Índice de Integridad Biológica (IBI) para peces y EPT (Ephemeroptera, Plecoptera, y Trichoptera) para la integridad de los ambientes acuáticos. Los resultados indican que en las zonas mejor conservadas se encuentran en los alrededores de Yurimaguas.

Palabras claves: Ictiofauna, biota acuática, Biodiversidad, Amazonia Peruana, conservación.

\section{Abstract}

The present paper describes the diversity and state of conservation, determinate by a rapid biological inventory, carried out since October 24 to 30 of 2005, at the Amazonian waterbodies from Northeast of Peru, between 680 and $133 \mathrm{~m}$ of altitude, among Tarapoto (San Martin) and Yurimaguas (Loreto). Data and samples were collected in 26 stations. Planckton samples were collected with standard net (40 microns), benthos with Surber net and fishes with small mesh seines. Descriptions of each habitat included coordinates (UTM), and limnological characteristics ( $\mathrm{pH}$, temperature, conductivity, oxygen). The richness of species of fishes was of 95 , dominated by Characiformes and Siluriformes. Richness of species in phytoplankton was of 74 , in zooplankton, 22 species and in benthos of 20 species. The conservation state was determined using the Index of Biological Integrity (IBI) for fishes, and EPT index (Ephemeroptera, Plecoptera, and Trichoptera) for the aquatic environments. The results indicate that in the better zones conserved were found in the around of Yurimaguas.

Keywords: Fishfauna, aquatic biota, Biodiversity, Peruvian Amazon, conservation.

\section{Introducción}

La Amazonia occidental (Alto Amazonas), está considerada como la zona con mayor diversidad y endemismo de peces, y es señalada como una región de alta prioridad para la conservación (Lowe-McConnell, 1987; Olson et al., 1998). En la Amazonia Peruana se conocen 750 especies de peces, de un total de 855 reportadas en todas las aguas continentales del Perú (Chang \& Ortega, 1995). Por otro lado, la actual diversidad de peces amazónicos podria incrementarse con recientes inventarios desarrollados en distintas regiones y ecosistemas, tal como los realizados en el Parque Nacional Manu (Ortega, 1996); la Zona Reservada Tambopata-Candamo (Chang, 1998); la Cuenca del río Yavarí (Ortega, datos no publicados); la Cuenca del río Madre de Dios (Barthem etal., 2003; Goulding et al., 2003). Sin embargo, la región nororiental ha sido muy poco estudiada, en particular los departamentos de Amazonas, Loreto y San Martín (Ortega \& Chang, 1998).

El presente trabajo describe la diversidad de peces, bentos y plancton y el estado de conservación de los ambientes acuáticos continentales en una zona de la región nororiental del Perú.

\section{Material y Métodos}

El estudio se realizó en parte de la cuenca hidrográfica del río Huallaga, entre Tarapoto (18M 0350523 y 9284466) en San Martin y Yurimaguas (18M 0382434 y 9344644) en Loreto (Fig. 1), ubicados entre los 133 y $382 \mathrm{~m}$ de altitud, respectivamente y registros de altura elevada de 600 - 680 m en San Roque, río Cumbaza y Cordillera La Escalera. El trabajo de campo fue desarrollado en octubre de 2005. El área específica del estudio comprendió parte de la cuenca del río Huallaga en Tarapoto, la Cordillera La Escalera, el valle del río Cainarache, tributarios del bajo Huallaga y el mismo río en Yurimaguas, siguiendo la carretera que une ambas ciudades y via fluvial desde Yurimaguas hasta laguna Sanango.

Los datos y muestras de peces provienen de 26 estaciones, divididos en 6 sectores: Tarapoto (5 estaciones), Cainarache (4 estaciones), Sabaloyacu (5 estaciones), Pongo (3 estaciones), Condoryacu-Pumayacu (4 estaciones) y Yurimaguas (5 estaciones). Las muestras de plancton y bentos fueron colectadas en ambientes represesntativos, uno por cada sector. El plancton fue colectado en tres ambientes lenticos (E19a, E19b, E20), y dos loticos (E2 y E5). Mientras que el bentos en cuatro ambientes lóticos (E1, E2, E3 y E4) del sector Cainarache.

En cada estacion se realizó la caracterizacion física del habitat (dimensiones del cuerpo de agua, tipo de agua, color aparente, profundidad) y el registro de parámetros limnológicos $(\mathrm{pH}$, tem- 
Tabla 1. Principales características y parámetros limnológicos por sectores, en el estudio de los cuerpos de agua Amazónicos en el nororiente del Perú. TARA= Tarapoto, CAIN= Cainarache, SABA= Sabaloyacu, PONG= Pongo, COPU= Cóndor-Pumayacu, YURI= Yurimaguas y STD $=$ Sólidos Totales Disueltos.

\begin{tabular}{lcccccc}
\hline Parámetro/Sector & TARA & CAIN & SABA & PONG & COPU & YURI \\
\hline Tipo de ambiente & lótico & lótico & lótico & lótico & lótico-léntico & lótico-léntico \\
Tipo de agua & clara-blanca & clara & clara & clara-blanca & blanca-negra & blanca-negra \\
Rango altitud (m) & $382-600$ & $500-680$ & $400-500$ & $290-240$ & $220-150$ & $140-133$ \\
Transparencia (m) & $0,1-0,5$ & $0,6-0,8$ & $0,6-0,9$ & $0,2-0,8$ & $0,1-0,5$ & $0,1-0,5$ \\
Tipo substrato & arena-roca & grava-roca & grava-roca & arena-arcilla & arena-arcilla & arena-arcilla \\
Velocidad agua & torrentosa & torrentosa & torrentosa & moderada & lenta & lenta \\
pH & $7,7-8,7$ & $6,6-10$ & $7,6-7,8$ & $7,7-8,1$ & $5,5-6,7$ & $7,1-7,6$ \\
Oxigeno disuelto (ppm) & $7,0,-7,5$ & $7,0-7,5$ & $7,0-7,6$ & $7,0-8,0$ & $2,5-7,1$ & $2,5-7,6$ \\
Conductividad (uS/cm) & $17-227$ & $25-209$ & $20-209$ & $29-739$ & $15-25$ & $55-225$ \\
STD $(\mathrm{ppm})$ & $19-219$ & $19-220$ & $11-116$ & $13-87$ & $14-90$ & $13-65$ \\
\hline
\end{tabular}

peratura, oxígeno disuelto, conductividad y sólidos totales disueltos) con un equipo multiparametros Hanna (Salcedo, 1998).

La colecta de peces se realizó utilizando redes $(5$ y $10 \mathrm{~m}$, malla $3-5 \mathrm{~mm}$ ) realizando cinco arrastres a la orilla en cada estación. El plancton fue colectado filtrando 50 litros de agua en una red estándar (malla de $40 \mu$ ). El bentos fue colectado con red Surber (malla 1 $\mathrm{mm}$ ), realizando tres replicas para obtener la densidad por metro cuadrado. La fijación del material biológico, peces y plancton, se realizó con formol 10 y $5 \%$, respectivamente y los organismos del bentos con etanol 70\%, según Ortega et al. (1998).

La separacion e identificacion se llevó a cabo en los laboratorios del Museo de Historia Natural. Las muestras fueron catalogadas y depositadas en el Museo de Historia Natural (Ortega et al., 1998; Fernández \& Domínguez, 2001).

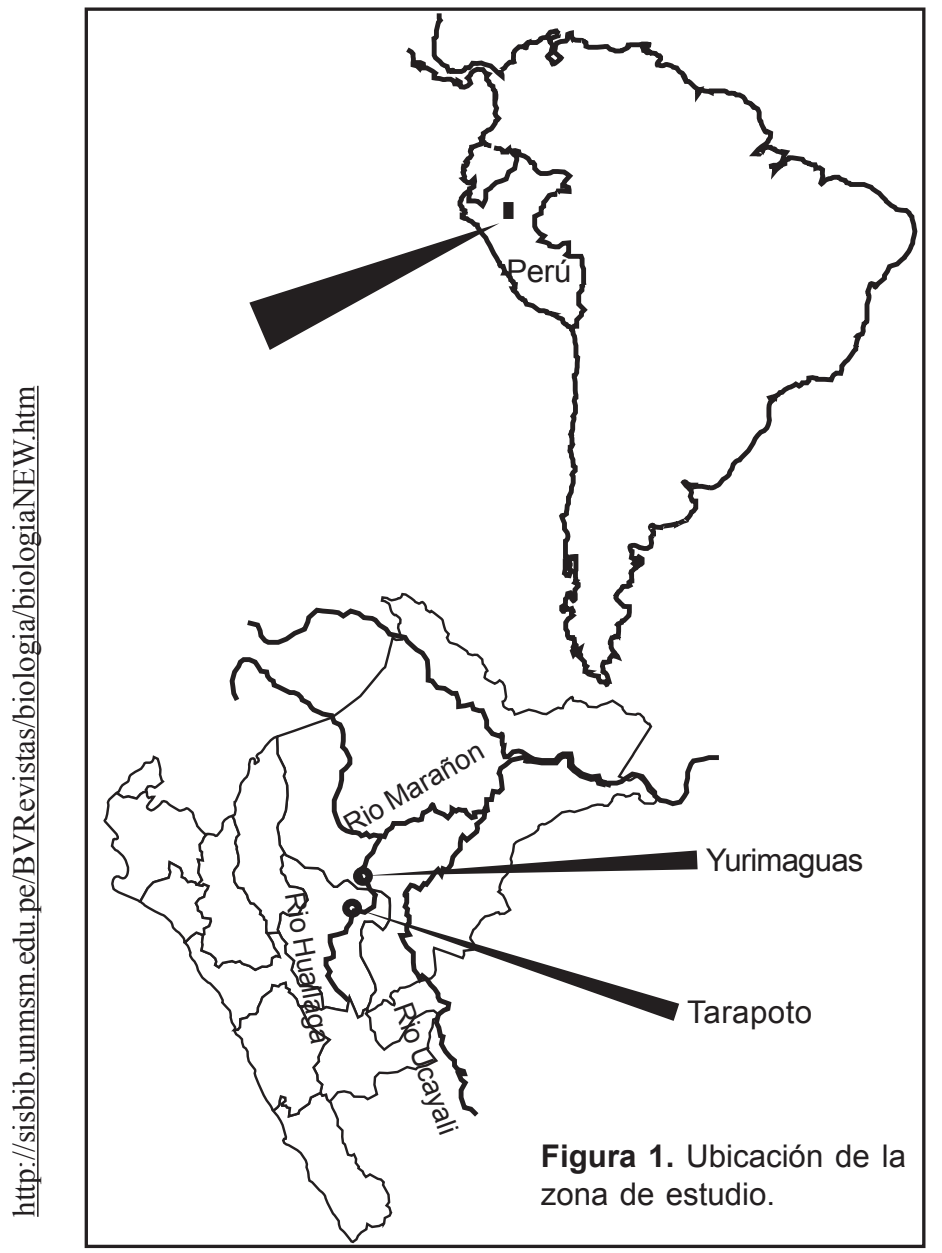

El estado de conservación de los ambientes acuáticos fue determinado mediante el Indice EPT (Ephemeroptera, Plecoptera, and Trichoptera) y el Indice de Integridad Biológica(IBI), basado en la comunidad de peces amazónicos.

El IBI es un sistema de calificación de hábitat diseñado por Karr \& Dudley (1981) y perfeccionado por Karr (1991), para evaluar la condición de cuerpos de agua en el hemisferio norte y basado en la comunidad de peces. El índice utiliza 12 medidas biológicas que reflejan la riqueza y composición de especies, estructura trófica, abundancia y condición de los peces. Un valor de 60 indica calidad prístina y 12 un fuerte impacto. Este índice fue adaptado y propuesto para analizar la condición de los ecosistemas peruanos basado en la comunidad de los peces amazónicos (Ortega et al., 2003b).

\section{Resultados y Discusión}

Los ambientes acuáticos evaluados corresponden a la cuenca del río Huallaga y comprende zonas de selva alta: San Roque y Cordillera La Escalera (Tarapoto) y selva baja (Pongo - Yurimaguas), las características de los habitats acuáticos y parámetros limnológicos difieren entre los sectores evaluados: aguas frías, torrentosas y de sustrato duro en los ríos y arroyos en los alrededores de Tarapoto, Cainarache, y Sabaloyacu; mientras que las aguas son más calmadas, el cauce más amplio y el sustrato blando y particulado como en los sectores del Pongo, Condoryacu-Pumayacu y Yurimaguas. En resumen observamos valores similares en oxigeno disuelto, transparencia y temperatura en los diferentes ambientes lóticos (Cainarache - Sabaloyacu) mientras, estos valores, fueron menores en los ambientes lénticos (Condoryacu-Pumayacu). Cabe destacar la existencia de valores altos de $\mathrm{pH}(>9)$ en la quebrada Cainarache de la Cordillera La Escalera; condición que podría explicar la dominancia de peces tolerantes (Rivulus, Cyprinodontidae) o secundarios, según Britski (1970). Un resumen de datos y rangos, por sectores en la Tabla 1 .

Tabla 2. Riqueza, como número de especies (S) del Fitoplancton, por taxa, en sectores representativos en el estudio de los cuerpos de agua Amazónicos en el nororiente del Perú. Cai $=$ Cainarache, Sab= Sabaloyacu, Pum= Pumayacu.

\begin{tabular}{lcccc}
\hline División & Familias & Cai & Sab & Pum \\
\hline Chlorophyta & 8 & 1 & 3 & 37 \\
Cyanophyta & 3 & & 1 & 9 \\
Bacillariophyta & 1 & 1 & 7 & 7 \\
Euglenophyta & 1 & 10 & & 12 \\
Total & $\mathbf{1 3}$ & $\mathbf{1 2}$ & $\mathbf{1 1}$ & $\mathbf{6 5}$ \\
\hline
\end{tabular}


Tabla 3. Riqueza (S) del bentos en sector Cainarache (E1E4) y Valores de EPT (N). Estudio de los cuerpos de agua Amazónicos en el nororiente del Perú.

\begin{tabular}{lccccc}
\hline Ordenes & Familias & CAI-E1 CAI-E2 & CAI-E3 CAI-E4 \\
\hline Odonata & 3 & & 1 & 1 & 1 \\
Plecoptera & 1 & 1 & 1 & 1 \\
Ephemeroptera & 2 & 1 & 2 & 1 & 1 \\
Trichoptera & 1 & & 2 & 2 & 1 \\
Coleoptera & 4 & & 2 & 4 & \\
Diptera & 2 & & 1 & 2 & \\
Otros & 4 & 2 & & 1 & 2 \\
Total & $\mathbf{1 7}$ & $\mathbf{3}$ & $\mathbf{9}$ & $\mathbf{1 1}$ & $\mathbf{6}$ \\
\hline EPT (E+P+T) & - & $\mathbf{1}$ & $\mathbf{1 9}$ & $\mathbf{1 1}$ & $\mathbf{1 7}$ \\
\hline
\end{tabular}

En el Fitoplancton fueron identificadas 74 especies de algas que corresponden a cuatro divisiones. En la riqueza (S), las Chlorophyta obtuvieron mayores cifras (37,50\%), siguen Euglenophyta (15, 21\%), Bacillariophyta (11,15\%) y Cyanophyta $(10,14 \%)$ ver Apéndice 1. Las aguas amazónicas pueden albergar una alta diversidad de organismos del plancton. La riqueza de algas es alta (Samanéz, 1979) en los ambientes lénticos de selva baja (llano amazónico) dependiendo de la cantidad de nutrientes, ausencia de corrientes y asociación a macrófitas acuáticas, siendo en cambio muy escasos en los ambientes lóticos ubicados a mayores altitudes (Esteves, 1998). Entre las microalgas, sobresalieron en riqueza las Chlorophyta (Desmidiaceae y Zygnemataceae, indicadoras de aguas ácidas), Roldan (1988). La abundancia de Chlorophyta, indica alta diversidad y cuerpos de agua con buenas condiciones, (concentración adecuada de nutrientes, $\mathrm{pH}$ neutro) según Branco (1978). En los cuerpos lénticos de Pumayacu se registraron 37 especies. Por otro lado, las Euglenophyta indican elevado contenido de materia orgánica (Roldan, 1992). Tabla 2.

En el Zooplancton, se identificaron 22 especies en cinco Phyla (Apéndice 2). En el total se evidencia la dominancia de Rotifera (11 spp.) y Protozoa (6 spp.) en aguas lénticas, en CóndorPumayacu y solo una especie (Nematode) en aguas torrentosas (Cainarache).

El bentos proviene de aguas loticas del sector de Cainarache, se identificaron 20 especies, 17 familias, 10 ordenes y dos Phyla: Arthropoda y Mollusca (Apéndice 3). La diversidad fue de baja a moderada (3 - 11 especies) en las diferentes estaciones, en su mayoría organismos de la clase Insecta. En la abundancia se registraron 78 individuos, destacando los Plecoptera (20) y Ephemeroptera (12), componentes del índice EPT. Los valores
Tabla 5. Distribución de la Riqueza (S) y Abundancia (N) por sectores. Estudio de los cuerpos de agua Amazónicos en el nororiente del Perú.

\begin{tabular}{lccccc}
\hline Sectores & Est. & $\mathbf{S}$ & $\mathbf{\%} \mathbf{S}$ & $\mathbf{N}$ & $\mathbf{\% N}$ \\
\hline Tarapoto & E22 & 4 & 4,44 & 23 & 1,09 \\
& E23 & 3 & 3,33 & 13 & 0,62 \\
& E24 & 5 & 5,56 & 197 & 9,35 \\
& E25 & 6 & 6,67 & 52 & 2,47 \\
Cainarache & E26 & 5 & 5,56 & 94 & 4,46 \\
& E1 & 2 & 2,22 & 60 & 2,85 \\
& E2 & 1 & 1,11 & 16 & 0,76 \\
Sabaloyacu & E3 & 6 & 6,67 & 64 & 3,04 \\
& E4 & 3 & 3,33 & 91 & 4,32 \\
& E5 & 1 & 1,11 & 8 & 0,38 \\
Pongo & E6 & 3 & 3,33 & 7 & 0,33 \\
& E7 & 2 & 2,22 & 5 & 0,24 \\
& E8 & 5 & 5,56 & 7 & 0,33 \\
Condoryacu- & E9 & 1 & 1,11 & 2 & 0,09 \\
Pumayacu & E10 & 9 & 10,00 & 28 & 1,33 \\
& E11 & 22 & 24,44 & 113 & 5,36 \\
& E12 & 16 & 17,78 & 191 & 9,06 \\
E18 & 7 & 7,78 & 177 & 8,40 \\
& E19 & 12 & 13,33 & 63 & 2,99 \\
& E20 & 9 & 10,00 & 184 & 8,73 \\
& E21 & 13 & 14,44 & 108 & 5,12 \\
& E13 & 12 & 13,33 & 142 & 6,74 \\
& E14 & 21 & 23,33 & 165 & 7,83 \\
& E15 & 19 & 21,11 & 103 & 4,89 \\
& E16 & 17 & 18,89 & 68 & 3,23 \\
& E17 & 9 & 10,00 & 127 & 6,02 \\
& $\mathbf{2 6}$ & $\mathbf{9 0}$ & $\mathbf{1 0 0}$ & $\mathbf{2 1 0 8}$ & $\mathbf{1 0 0}$ \\
\hline
\end{tabular}

mayores de EPT se registraron en las estaciones E2, E4 y E3 (quebradas) y muy baja en E1 (río Cainarachi). Tabla 3.

El mayor número de especies de los órdenes Ephemeroptera, Plecoptera y Trichoptera indican cuerpos de agua de buena calidad, en cambio su escasez y el incremento de la abundancia de Diptera (Chironomidae) y Annelida implica cuerpos de agua deteriorados. La determinación de la calidad del agua esta en relación a la proporción abundancia en que estos grupos se encuentren representados en la comunidad (Roldan, 1988).

Los peces presentaron una riqueza de 95 especies pertenecientes a 23 familias y 8 órdenes y una abundancia total de 2113 individuos, registrados en seis sectores (26 estaciones) entre Tarapoto

Tabla 4. Resumen de Riqueza (S) de peces por Órdenes, familias y sectores, en el estudio de los cuerpos de agua Amazónicos en el nororiente del Perú. .TARA=Tarapoto, CAIN=Cainarache, SABA=Sabaloyacu, PONG=Pongo, COPU=CondorPumayacu, YURI= Yurimaguas.

\begin{tabular}{|c|c|c|c|c|c|c|c|}
\hline \multirow[t]{2}{*}{ ORDENES } & \multirow[t]{2}{*}{ Familias } & \multicolumn{5}{|c|}{ No. Especies } & \multirow[b]{2}{*}{ YURI } \\
\hline & & TARA & CAIN & SABA & PONG & COPU & \\
\hline Clupeiformes & 1 & & & & 1 & & 1 \\
\hline Characiformes & 9 & 7 & 3 & 6 & 25 & 20 & 39 \\
\hline Gymnotiformes & 2 & & & & & & 2 \\
\hline Siluriformes & 5 & 4 & 3 & 1 & 8 & 3 & 8 \\
\hline Atheriniformes & 1 & 1 & 1 & 1 & & 1 & 2 \\
\hline Cyprinodontiformes & 2 & 2 & & & 3 & & \\
\hline Synbranchiformes & 1 & & 1 & & & & \\
\hline Perciformes & 2 & 2 & & & 3 & 6 & 4 \\
\hline Totales & 23 & 16 & 8 & 8 & 40 & 30 & 56 \\
\hline
\end{tabular}




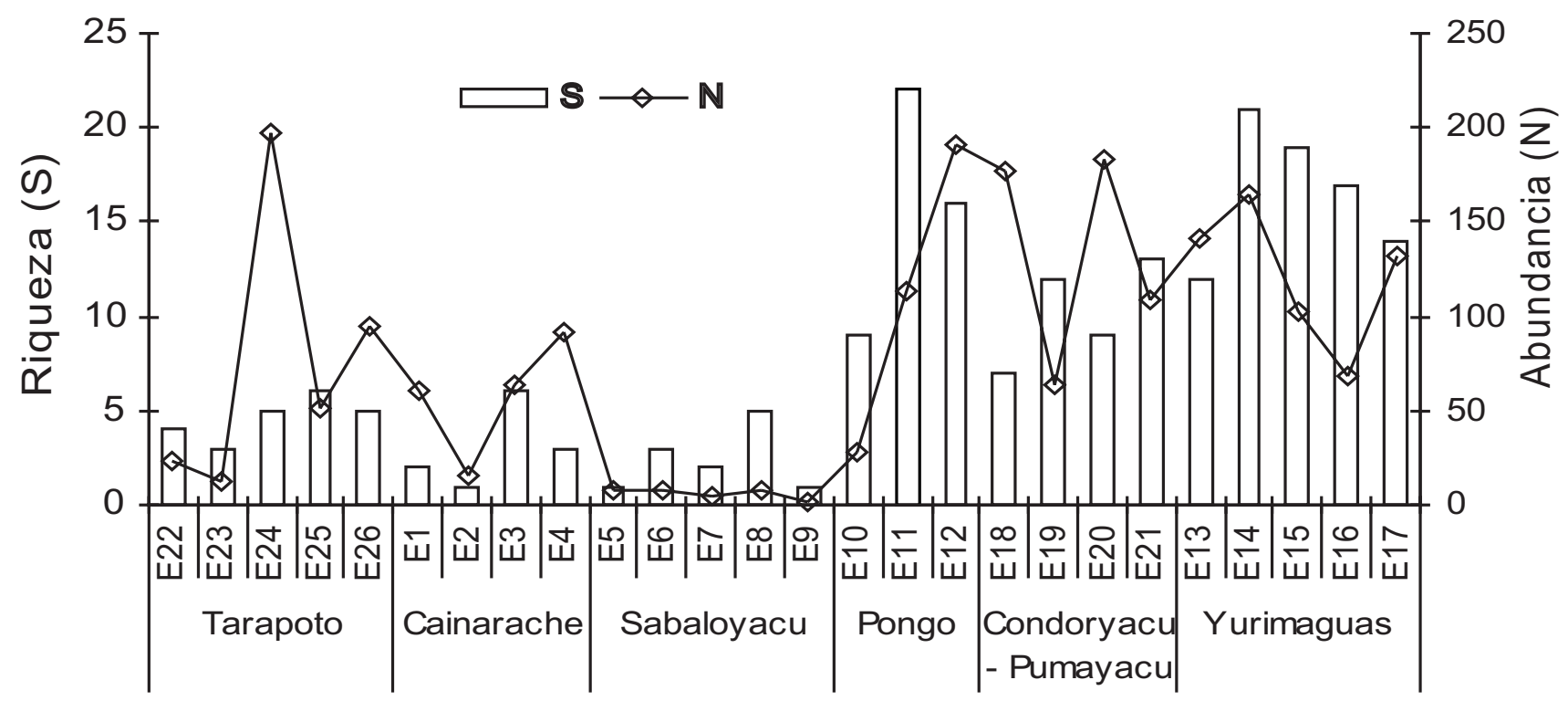

Figura 2. Riqueza (S) y Abundancia (N) de peces, en el estudio de los cuerpos de agua Amazónicos en el nororiente del Perú.

y Yurimaguas (Apéndice 4). El orden Characiformes presenta la mayor riqueza $(58,62 \%)$, siguen Siluriformes $(19,20 \%)$ y Perciformes $(10,11 \%)$. Estos resultados coinciden con el patrón de distribución observado en la región amazónica y neotropical por Ortega (1996), Goulding et al. (2003) y Lowe-McConnell (1987).

La mayor riqueza de peces se encontró en Yurimaguas (menor altitud y condiciones favorables) y la menor en Cainarache y Sabaloyacu, cerca a Tarapoto (mayor altitud) (Tablas 4 y 5, Fig. 2). La abundancia por órdenes también esta dominada por los Characiformes (62\%), siguen Siluriformes (16\%) y Perciformes $(11 \%)$ y los demás con menos del $1 \%$. Considerando la riqueza por familias, destacan los Characidae (46,3\%), constituidos por indiviuos pequeños que frecuentan las orillas de los ambientes lóticos, siguen Cichlidae (9,5\%) y Loricariidae (8,4\%); las otras 18 familias presentan menos del 5\%. Los Characidae fueron también los más numerosos (74,6\%), seguidos de los Cichlidae (11,6\%) Tabla 6. Observando la composición de especies por sectores evaluados, en la parte baja (Yurimaguas) se registró el mayor porcen-

Tabla 6. Riqueza (S) y abundancia (N) por famílias de peces, en el estudio de los cuerpos de agua Amazónicos en el nororiente del Perú.

\begin{tabular}{lcccc}
\hline Familias & $\mathbf{S}$ & $\mathbf{\%} \mathbf{S}$ & $\mathbf{N}$ & $\mathbf{0} \mathbf{N}$ \\
\hline Anostomidae & 1 & 1,05 & 5 & 0,24 \\
Apteronotidae & 1 & 1,05 & 1 & 0,05 \\
Aspredinidae & 1 & 1,05 & 1 & 0,05 \\
Belonidae & 2 & 2,11 & 3 & 0,14 \\
Characidae & 44 & 46,32 & 1577 & 74,63 \\
Cichlidae & 9 & 9,47 & 246 & 11,64 \\
Crenuchidae & 4 & 4,21 & 26 & 1,23 \\
Curimatidae & 4 & 4,21 & 32 & 1,51 \\
Engraulididae & 1 & 1,05 & 6 & 0,28 \\
Erythrinidae & 1 & 1,05 & 3 & 0,14 \\
Acestrorhynchidae & 1 & 1,05 & 2 & 0,09 \\
Hemiodontidae & 1 & 1,05 & 1 & 0,05 \\
Heptapteridae & 4 & 4,21 & 24 & 1,14 \\
Lebiasinidae & 1 & 1,05 & 42 & 1,99 \\
Loricariidae & 8 & 8,42 & 70 & 3,31 \\
Pimelodidae & 4 & 4,21 & 4 & 0,19 \\
Poecilidae & 1 & 1,05 & 1 & 0,05 \\
Prochilodontidae & 1 & 1,05 & 1 & 0,05 \\
Rhamphichthyidae & 1 & 1,05 & 1 & 0,05 \\
Rivulidae & 1 & 1,05 & 57 & 2,70 \\
Sciaenidae & 1 & 1,05 & 1 & 0,05 \\
Synbranchidae & 1 & 1,05 & 1 & 0,05 \\
Trichomycteridae & 2 & 2,11 & 8 & 0,38 \\
23 & $\mathbf{9 5}$ & $\mathbf{1 0 0}$ & $\mathbf{2 1 1 3}$ & $\mathbf{1 0 0}$ \\
\hline & & & & \\
\hline
\end{tabular}

taje (36), sigue el sector del Pongo (25) y con menores valores los sectores de Cainarache y Sabaloyacu (Tabla 4).

En relación al Índice de Integridad Biológica (IBI), los valores obtenidos tienen relación directa con los resultados de riqueza $(\mathrm{S})$ y abundancia $(\mathrm{N})$, los mayores valores se presentaron en los sectores de selva baja (Yurimaguas, Condoryacu-Pumayacu y Pongo) y los menores en los sectores de selva alta (Cainarache, Sabaloyacu). Valores altos del IBI indican usualmente comunidades maduras y diversas, mientras que valores mínimos caracterizan a los ambientes alterados o afectados por diversas actividades humanas (Ortega et al., 2003a)(ver Tabla 7). El Índice de Integridad Biológica muestra que los cuerpos de agua con buenas condiciones abióticas y menor intervención humana se presentan en selva baja, entre Pongo y Yurimaguas, donde también se presentaron mayor riqueza y abundancia. Estos ambientes se caracterizaron por presentar una corriente con remansos, recodos, orillas amplias, troncos apilados, hojarasca y ramas caídas, constituyendo un mejor hábitat para el desarrollo de las diversas comunidades acuáticas, lo cual se ve favorecido por la presencia de mayor cobertura vegetal, la proveería de alimento y de sombra que regularia la temperatura del agua y protegeria a la orilla de erosión (Allan, 1995).

Considerando el bentos, la calidad del agua puede ser considerada entre aceptable y buena; sin embargo, se requiere la inclusión de mayor número de estaciones y la continuidad del estudio.

La mayor riqueza y abundancia de peces en los alrededores de Yurimaguas que en la zona de Tarapoto, coincide con la variedad de habitats y recursos que presenta el primero. Por otro lado, algunas estaciones de Cainarache, se encuentran en un estado desfavorable, asociado al inadecuado uso de los ambientes acuáticos. Cabe mencionar que en esta evaluación en Cainarache no fueron colectadas dos especies de Astroblepidae registrados en 1984 por nosotros (datos no publicados)

\section{Agradecimientos}

Al CSI, ICBAR, Museo de Historia Natural (UNMSM) y al Dr. S. Schaefer (American Museum of Natural History) por el auspicio y colaboración, respectivamente; para el desarrollo del estudio y a los colaboradores locales por el apoyo recibido durante el trabajo de campo. 
Tabla 7. Valores del Índice de Integridad Biológica (IBI); en el estudio de los cuerpos de agua Amazónicos en el nororiente del Perú. TARA=Tarapoto, CAIN=Cainarache, SABA=Sabaloyacu, PONG=Pongo, COPU=Condor-Pumayacu y YURI=Yurimaguas.

\begin{tabular}{|c|c|c|c|c|c|c|c|c|}
\hline Categoría & Métrica & Criterio & TARA & CAIN & SABA & PONG & COPU & YURI \\
\hline \multirow{6}{*}{$\begin{array}{l}\text { Riqueza o } \\
\text { diversidad } \\
\text { de especies }\end{array}$} & 1 & Cantidad especies & 3 & 1 & 1 & 3 & 3 & 5 \\
\hline & 2 & Characiformes & 3 & 3 & 3 & 5 & 5 & 5 \\
\hline & 3 & Siluriformes & 3 & 3 & 1 & 5 & 3 & 5 \\
\hline & 4 & Gymnotiformes & 1 & 1 & 1 & 3 & 1 & 1 \\
\hline & 5 & Otros & 1 & 1 & 1 & 3 & 5 & 5 \\
\hline & 6 & Tolerantes & 1 & 1 & 1 & 3 & 5 & 5 \\
\hline \multirow{3}{*}{$\begin{array}{l}\text { Composición } \\
\text { trófica de las } \\
\text { especies }\end{array}$} & 7 & Omnívoros & 3 & 3 & 3 & 5 & 3 & 3 \\
\hline & 8 & Detritívoros & 3 & 3 & 3 & 5 & 3 & 5 \\
\hline & 9 & Carnívoros & 1 & 1 & 1 & 5 & 5 & 5 \\
\hline \multirow{4}{*}{$\begin{array}{l}\text { Abundancia y } \\
\text { condición de } \\
\text { los peces }\end{array}$} & 10 & No. individuos & 5 & 5 & 3 & 3 & 3 & 1 \\
\hline & 11 & Saludables & 5 & 5 & 5 & 5 & 5 & 5 \\
\hline & 12 & Lesionados & 5 & 5 & 5 & 5 & 5 & 5 \\
\hline & & TOTAL & 35 & 32 & 28 & 50 & 46 & 50 \\
\hline
\end{tabular}

\section{Literatura citada}

Allan, D. 1995. Stream Ecology, structure and function of running waters. Chapman and Hall, 2-6 Boundary Row, London. 388 pp.

Barthem, R., M. Goulding, B. Forsberg, C. Cañas, H. Ortega. 2003. Aquatic Ecology of the río Madre de Dios. ACA \& ACCA. Grafica Biblos. Lima, Peru. 117 pp.

Britski, H. 1970. Peixes de agua doce do Estado de são PauloSistematica. In: Com. Interest. Da Bacia Paraná-Uruguai. Poluicao e Piscicultura São Paulo, pp. 79-112.

Branco, S . 1978. Hidrobiología Aplicada a Engenharia Sanitaria. 2ed. Sao Paulo (CETESB).

Chang, F., 1998. The fishes of the Tambopata - Candamo Reserved Zone, Southeastern Peru. Revista Peruana de Biologia Vol 5 (1): 17-26.

Esteves, F. 1998. Limnología Tropical. Editorial Interciencia. río de Janeiro. Brasil. 350 pp.

Fernández, H.R. y E. Domínguez. 2001. Guía para la Determinación de Artrópodos Bentónicos Sudamericanos. Serie: Investigaciones de la UNT, Sub serie Ciencias Exactas Naturales. Tucumán. Argentina. 282 pp.

Goulding, M., C. Cañas, R. Barthem, B. Fosberg, H. Ortega. 2003. Amazon Headwaters. Rivers, Wildlife and Conservation in Sotheastern Peru. ACA \& ACCA. Grafica Biblos. Lima, Peru. 198 pp.

Lowe-McConnell, R. 1987. Ecological Studies in Tropical Fish Communities. Cambridge Univ. Press, 382 pp.

Karr, J.R. 1991. Biology Integrity: a long - neglected aspect of water resource management. Ecological Applications 1:66-84.

Karr, J.R. and Dudley, D.R. 1981. Ecological perspective on water quality goals. Environmental Management 5:55-68.
Merrit, R.W. \& K.W. Cummins. 1996. Aquatic insects of North América. Kendall/Hunt Publishing Company, Duque, Iowa. USA. $862 \mathrm{pp}$.

Olson, D., E. Dinerstein, P. Canevari, I. Davidson, G. Castro, V. Morisset, R. Abell and E. Toledo. 1998. Freshwater Biodiversity of Latin America and the Carbbean. A Conservation Assessment. Biodiversity Support Program, Washington, DC. 70 pp.

Ortega, H. 1996. Ictiofauna del Parque Nacional Manu, MD, Perú. En: Wilson \& Sandoval: MANU: La Biodiversidad en el Sureste del Perú. Smithsonian Institution, Washington D.C.

Ortega, H., I. Samanez, E. Castro, M. Hidalgo y N. Salcedo. 1998. Protocolos Sugeridos para la Evaluación y Monitoreo de Sistemas Acuáticos del Bajo Urubamba, Perú. En: Dallmeier, F. Biodiversity Assessment \& Monitoring, Smithsonian Institution/MAB Series \#2: 278-280.

Ortega H., \& F. Chang. 1998. Peces Continentales del Perú. En Diversidad Biológica en Iberoamérica. Vol III. Ed. G. Halfter, Mexico.

Ortega, H., M. Hidalgo \& G. Bertiz. 2003a. Los Peces del río Yavarí. En: Pitman, N., C. Vriesendorp, D. Moskovits (Eds.) YAVARI: Rapid Biological Inventories Report 11. Chicago IL: The Field Museum. Pp: 59-62 y 220-243.

Roldan, G. 1988. Guía para el estudio de los macro invertebrados del departamento de Antioquia. Fondo FEN- Colombia, Ed. Presencia Ltda. Bogota. 217 pp.

Roldan, G. 1992. Fundamentos de Limnología Neotropical. Universidad de Antioquia. Colombia. 235 pp.

Samanéz, I. 1979. Algas continentales del Perú II. Algas de Pucallpa y alrededores. Serie de Divulgación No.10, Museo de Historia Natural, UNMSM. 42 pp. y 115 figs.

Apéndice 1. Composición taxonómica del fitoplacton en el estudio de los cuerpos de agua Amazónicos en el nororiente del Perú.

\begin{tabular}{|c|c|c|c|c|c|c|c|}
\hline División & Familia & Especies & E19 a & E19 b & E20 & E2 & E-5 \\
\hline \multirow[t]{11}{*}{ CHLOROPHYTA } & & Closterium dianae & & $x x$ & $x x x$ & & \\
\hline & & Closterium libellula & $x x$ & $x x$ & $\mathrm{xx}$ & & \\
\hline & & Closterium malmei & $x x$ & $x x$ & & & \\
\hline & & Closterium setaceum & $x x$ & & & & \\
\hline & & Cosmarium circulare & $x x$ & $x x$ & $x \mathrm{x}$ & & \\
\hline & & Cosmarium reniforme & $x x$ & $x x$ & $x x$ & & \\
\hline & & Cosmarium sp 1 & $x x$ & $x x$ & & & \\
\hline & & Cosmarium sp 2 & $x x$ & $x x$ & & & \\
\hline & & Cosmarium sp 3 & $x x$ & & $x x$ & & \\
\hline & & Desmidium aequale & & & $x x$ & & \\
\hline & & Desmidium cylindricum & & & $x x x$ & & \\
\hline
\end{tabular}


Apéndice 1. Continuación

\begin{tabular}{|c|c|c|c|c|c|c|c|}
\hline División & Familia & Especies & E19 a & E19 b & E20 & E2 & E-5 \\
\hline & 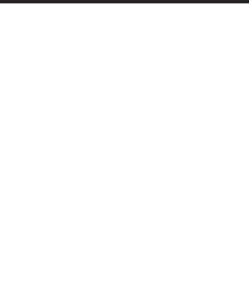 & $\begin{array}{l}\text { Desmidium graciliceps } \\
\text { Desmidium quadratum } \\
\text { Desmidium swartzii } \\
\text { Euastrum ansatum } \\
\text { Euastrum gemmatum } \\
\text { Euastrum spinulosum } \\
\text { Gonatozygon monotaenium } \\
\text { Micrasterias borgei } \\
\text { Micrasterias laticeps } \\
\text { Micrasterias mahabuleshwarensis }\end{array}$ & $\begin{array}{l}x x \\
x x \\
x x \\
x x\end{array}$ & $\begin{array}{l}x x \\
x x \\
x x x \\
x x \\
x x \\
x x\end{array}$ & $\begin{array}{l}x x \\
x x \\
x x\end{array}$ & & \\
\hline & Desmidiaceae & Netrium digitus & & & $x x$ & & \\
\hline & Oedogoniaceae & Oedogonium sp & $x x$ & $x x$ & & $x x$ & \\
\hline & Palmellaceae & Palmella mucosa & $x x$ & & & & \\
\hline & Hydrodictyaceae & Pediastrum duplex var. clathratum & $x x$ & $x x$ & & & \\
\hline & Cladophoraceae & Rhizoclonium crassipellitum & $x x$ & & & & \\
\hline & Scenedesmaceae & $\begin{array}{l}\text { Scenedesmus alternans } \\
\text { Scenedesmus bijuga }\end{array}$ & & $\begin{array}{l}x x \\
x x\end{array}$ & & & \\
\hline & Zygnemataceae & $\begin{array}{l}\text { Spirogyra sp } 1 \\
\text { Spirogyra } s p 2 \\
\text { Mougeotia } s p \\
\text { Spirogyra } s p 3\end{array}$ & $\begin{array}{l}x x \\
x x \\
x x \\
x x\end{array}$ & $x x$ & $\begin{array}{l}x x x \\
x x \\
x x\end{array}$ & & $\begin{array}{l}x \times x \\
x x x \\
x x x\end{array}$ \\
\hline & Desmidiaceae & $\begin{array}{l}\text { Staurastrum muticum } \\
\text { Staurastrum rotula } \\
\text { Staurastrum zonata }\end{array}$ & $x x$ & $\begin{array}{l}x x \\
x x\end{array}$ & & & \\
\hline & Ulothricaeae & $\begin{array}{l}\text { Ulothrix cylindricum } \\
\text { Ulothrix zonata }\end{array}$ & $x x$ & $\begin{array}{l}x x \\
x x\end{array}$ & $x x$ & & \\
\hline CYANOPHYTA & $\begin{array}{l}\text { Nostocaceae } \\
\text { Chroococcaceae }\end{array}$ & $\begin{array}{l}\text { Anabaena circinalis } \\
\text { Coelosphaerium dubium } \\
\text { Merismopedia glauca }\end{array}$ & $x x$ & & $x x$ & & \\
\hline & Oscillatoriaceae & $\begin{array}{l}\text { Lyngbya aestuari } \\
\text { Oscillatoria chalybea } \\
\text { Oscillatoria formosa } \\
\text { Oscillatoria limosa } \\
\text { Oscillatoria ornata } \\
\text { Oscillatoria princeps } \\
\text { Oscillatoria tenuis } \\
\text { Spirulina princeps }\end{array}$ & $\begin{array}{l}x x \\
x x \\
x x \\
x x \\
x x \\
x x \\
x x\end{array}$ & $x \times x$ & $x x$ & & $x \times x$ \\
\hline BACILLARIOPHYTA & Bacillariaceae & $\begin{array}{l}\text { Cymbella turgida } \\
\text { Eunotia triodon } \\
\text { Fragillaria sp } \\
\text { Gomphonema olivaceum } \\
\text { Navicula criptocephala } \\
\text { Navicula mutica } \\
\text { Nitzschia linearis } \\
\text { Nitzschia sigmoidea } \\
\text { Pinnularia grandis } \\
\text { Pinnularia microstauron } \\
\text { Tabellaria sp }\end{array}$ & $\begin{array}{l}x x \\
x x \\
\\
x x \\
x x \\
x x\end{array}$ & & $\begin{array}{l}x \times x \\
x x \\
x x x\end{array}$ & $x x$ & $\begin{array}{l}x x x \\
x x \\
x x x \\
x x x \\
x x x \\
x x \\
x x\end{array}$ \\
\hline EUGLENOPHYTA & Euglenaceae & $\begin{array}{l}\text { Euglena acus } \\
\text { Euglena ehrenbergii } \\
\text { Euglena spirogyra } \\
\text { Euglena tripteris } \\
\text { Lepocinclis ovum } \\
\text { Phacus curvicauda } \\
\text { Phacus longicauda } \\
\text { Phacus orbicularis } \\
\text { Phacus tortus } \\
\text { Strombomonas sp } \\
\text { Trachelomonas armata } \\
\text { Trachelomonas cylindrica } \\
\text { Trachelomonas hispida } \\
\text { Trachelomonas superba } \\
\text { Trachelomonas volvocina } \\
74\end{array}$ & $\begin{array}{l}x x \\
x x \\
x x \\
x x \\
x x \\
x x \\
x x \\
x x\end{array}$ & $\begin{array}{l}x x \\
x x \\
x x\end{array}$ & $\begin{array}{l}x x \\
22\end{array}$ & $\begin{array}{l}x x x \\
x x x \\
x x x \\
x x x \\
x x x \\
x x x \\
x x \\
x x \\
x x \\
x x x \\
12\end{array}$ & 11 \\
\hline
\end{tabular}

Apéndice 2. Composición taxonómica del zooplacton en el estudio de los cuerpos de agua Amazónicos en el nororiente del Perú.

\begin{tabular}{|c|c|c|c|c|c|c|}
\hline Clase & Especie & E19 a & E19 b & E20 & E2 & E-5 \\
\hline Rotifera & $\begin{array}{l}\text { Dipleuchlanis propatula } \\
\text { Colurella adriatica } \\
\text { Epiphanes macrourus } \\
\text { Lecane bulla } \\
\text { Lecane curvicornis }\end{array}$ & $\begin{array}{l}x x \\
x x \\
x x \\
x x\end{array}$ & $\begin{array}{l}x x \\
x x\end{array}$ & & & Continúa... \\
\hline
\end{tabular}


Apéndice 2. Continuación

\begin{tabular}{|c|c|c|c|c|c|c|}
\hline Clase & Especie & E19 a & E19b & E20 & E2 & E-5 \\
\hline & Lecane hamata & $\mathrm{xx}$ & & & & \\
\hline & Lecane leontina & $x x$ & & & & \\
\hline & Lepadella ovalis & $x x$ & & & & \\
\hline & Platyias leloupi & & $\mathrm{x}$ & & & \\
\hline & Platyonus patulus & & $\mathrm{x}$ & & & \\
\hline & Sinantherina semibullata & $x x$ & & & & \\
\hline & Sinantherina spinosa & $x x x$ & $x x$ & & & \\
\hline & Testudinella patina & $x x$ & & & & \\
\hline & Trichotria tetractis & $x x$ & & & & \\
\hline Cladocera & Moina micrura & & $x x$ & & & \\
\hline Copepoda & Mesocyclops longicauda & & $\mathrm{x}$ & & & \\
\hline \multirow[t]{6}{*}{ Protozoa } & Arcella vulgaris & $x x$ & & $x x$ & & \\
\hline & Arcella sp 1 & $x x$ & & $x x$ & & \\
\hline & Arcella sp 2 & $x x$ & & $x x$ & & \\
\hline & Difflugia sp & $x x$ & & & & \\
\hline & Vorticella $s p$ & $x x$ & & $x x$ & & \\
\hline & Euglipha acanthophora & $\mathrm{xx}$ & & & & \\
\hline \multirow[t]{2}{*}{ Nematoda } & Nematode n.i & $\mathrm{xx}$ & & & $x x$ & \\
\hline & Total por estacion & 18 & 7 & 4 & 1 & 0 \\
\hline
\end{tabular}

Apéndice 3. Composición taxonómica del bentos en el estudio de los cuerpos de agua Amazónicos en el nororiente del Perú.

\begin{tabular}{|c|c|c|c|c|c|c|c|c|}
\hline Orden & Familia & Especie & CAI-E1 & CAI-E2 & CAI-E3 & CAI-E4 & Total & $\begin{array}{l}\text { Abundancia } \\
\text { relativa }(\%)\end{array}$ \\
\hline Lepidoptera & Pyralidae & Petrophila sp. & & & 3 & & 3 & 3,8 \\
\hline \multirow[t]{3}{*}{ Odonata } & Coenagrionidae & Acanthagrion sp. & & & & 1 & 1 & 1,3 \\
\hline & Libellulidae & Dythemis sp. & & & 1 & & 1 & 1,3 \\
\hline & Polythoridae & Polythore sp. & & 2 & & & 2 & 2,6 \\
\hline Megaloptera & Corydalidae & Corydalus sp. & & & 1 & 1 & 2 & 2,6 \\
\hline Plecoptera & Perlidae & Anacroneuria sp. & & 6 & 7 & 7 & 20 & 25,6 \\
\hline \multirow[t]{2}{*}{ Ephemeroptera } & Baetidae & Baetis sp. & & 1 & & & 1 & 1,3 \\
\hline & Leptophlebiidae & Thraulodes sp. & 1 & 1 & 2 & 8 & 12 & 15,4 \\
\hline \multirow[t]{2}{*}{ Trichoptera } & Hydropsychidae & Macrosternum sp. & & 9 & & & 9 & 11,5 \\
\hline & & Smicridea $s p$. & & 2 & 2 & 2 & 6 & 7,7 \\
\hline \multirow[t]{2}{*}{ Hemiptera } & Naucoridae & Cryphocricos sp. & & & & 1 & 1 & 1,3 \\
\hline & & Limnocoris sp. & 1 & & & & 1 & 1,3 \\
\hline \multirow[t]{5}{*}{ Coleoptera } & Dryopidae & Pelonomus sp. & & & 1 & & 1 & 1,3 \\
\hline & Elmidae & Disersus sp. & & 3 & & & 3 & 3,8 \\
\hline & & Heterelmis sp. & & & 3 & & 3 & 3,8 \\
\hline & Ptilodactylidae & Anchytarsus sp. & & 2 & 2 & & 4 & 5,1 \\
\hline & Psephenidae & Psepehenus sp. & & & 1 & & 1 & 1,3 \\
\hline \multirow[t]{2}{*}{ Diptera } & Chironomidae & Chironomidae & & & 2 & & 2 & 2,6 \\
\hline & Tipulidae & Hexatoma $s p$. & & 3 & & & 3 & 3,8 \\
\hline \multirow[t]{6}{*}{ Basommatophora } & Basommatidae & sp. 1 & 2 & & & & 2 & 2,6 \\
\hline & & No. Individuos $(\mathrm{N})$ & 4 & 29 & 25 & 20 & 78 & 100 \\
\hline & & No. Especies (S) & 3 & 9 & 11 & 6 & 20 & - \\
\hline & & Diversidad $\mathrm{H}^{\prime}$ & 1,50 & 2,80 & 3,16 & 2,04 & & - \\
\hline & & Indice de Margalef & 1,44 & 2,38 & 3,11 & 1,67 & - & - \\
\hline & & EPT & 1 & 19 & 11 & 17 & & \\
\hline
\end{tabular}

Apéndice 4. Composición taxonómica de peces en el estudio de los cuerpos de agua Amazónicos en el nororiente del Perú.

\begin{tabular}{|c|c|c|c|c|c|c|c|c|}
\hline Orden / Familia / Especie & TARA & CAIN & SABA & PONG & COPU & YURI & Total & $\%$ \\
\hline \multicolumn{9}{|l|}{ CLUPEIFORMES } \\
\hline \multirow{2}{*}{\multicolumn{9}{|c|}{$\begin{array}{l}\text { Anchoviella sp. } \\
\text { CHARACIFORMES }\end{array}$}} \\
\hline & & & & & & & & \\
\hline \multicolumn{9}{|l|}{ Acestrorhynchidae } \\
\hline Acestrorhynchus sp. & & & & 1 & 1 & & 2 & 0,09 \\
\hline \multicolumn{9}{|l|}{ Characidae } \\
\hline Aphyocharax sp. & & & & & & 10 & 10 & 0,47 \\
\hline Aphyochrax pusillus & & & & 4 & & 15 & 19 & 0,90 \\
\hline Astyanax bimaculatus & 6 & & & 1 & 13 & 18 & 38 & 1,80 \\
\hline Astyanax maximus & & & 1 & 3 & 1 & 1 & 6 & 0,28 \\
\hline Astyanax sp. & & & & & 2 & 1 & 3 & 0,14 \\
\hline Bryconamericus sp. & 1 & 8 & & & & & 9 & 0,43 \\
\hline Ceratobranchia sp. & 52 & & & & & & 52 & 2,46 \\
\hline Charax sp. & & & & & 9 & & 9 & 0,43 \\
\hline Cheirodontinae (alevino) & & & & & & 1 & 1 & 0,05 \\
\hline Clupeocharax anchoveoides & & & & & & 11 & 11 & 0,52 \\
\hline Creagrutus ortegai & 51 & 59 & 1 & 43 & 3 & 57 & 214 & 10,13 \\
\hline Creagrutus sp. 1 & & & & 26 & & & 26 & 1,23 \\
\hline Creagrutus sp. 2 & & & & 38 & & & 38 & 1,80 \\
\hline Ctenobrycon spilurus & & & & & 3 & 12 & 15 & 0,71 \\
\hline Engraulisoma taeniatum & & & & & & 1 & 1 & 0,05 \\
\hline \multirow[t]{2}{*}{ Galeocharax gulo } & & & & & & 1 & 1 & 0,05 \\
\hline & & & & & & & \multicolumn{2}{|c|}{ Continúa... } \\
\hline
\end{tabular}


Apéndice 4. Continuación.

\begin{tabular}{|c|c|c|c|c|c|c|c|c|}
\hline Orden / Familia / Especie & TARA & CAIN & SABA & PONG & COPU & YURI & Total & $\%$ \\
\hline Galeocharax sp. & & & & 1 & & 8 & 9 & 0,43 \\
\hline Gnatocharax sp. & & & & & & 1 & 1 & 0,05 \\
\hline Gymnocorymbus sp. & & & & & & 2 & 2 & 0,09 \\
\hline Hemibrycon sp. & 1 & & 3 & 1 & & & 5 & 0,24 \\
\hline Hemigrammus sp. 1 & & & & 1 & 17 & & 18 & 0,85 \\
\hline Hemigrammus sp. 2 & & & & 1 & 1 & & 2 & 0,09 \\
\hline Holoshestes sp. & & & & 8 & & 8 & 16 & 0,76 \\
\hline Hyphessobrycon eritrostigma & & & & 2 & & 5 & 7 & 0,33 \\
\hline Hyphessobrycon sp. & & & & 2 & & & 2 & 0,09 \\
\hline Knodus sp. 1 & & & & 2 & & 15 & 17 & 0,80 \\
\hline Knodus sp. 2 & & & & 8 & & 18 & 26 & 1,23 \\
\hline Knodus sp. 3 (caudal rojita) & 166 & & & & & & 166 & 7,86 \\
\hline Knodus sp. 4 & 41 & 139 & & 70 & 207 & 204 & 661 & 31,28 \\
\hline Leptagoniates steindachneri & & & & & & 1 & 1 & 0,05 \\
\hline Moenkhausia dichroura & & & & & & 16 & 16 & 0,76 \\
\hline Moenkhausia oligolepis & & & & 6 & 2 & 57 & 65 & 3,08 \\
\hline Odontostilbe sp. & & & & & 2 & 4 & 6 & 0,28 \\
\hline Parecbasis $s p$. & & & & & & 2 & 2 & 0,09 \\
\hline Phenacogaster sp. & & & & 1 & & 13 & 14 & 0,66 \\
\hline Prionobrama filigera & & & & & & 23 & 23 & 1,09 \\
\hline Prodontocaharax sp. & & & & 4 & & 11 & 15 & 0,71 \\
\hline Roeboides sp. & & & & & & 1 & 1 & 0,05 \\
\hline Scopaeocharax rhinodus & & & & 8 & & 1 & 9 & 0,43 \\
\hline Serrapinus heterodon & & & & 10 & & & 10 & 0,47 \\
\hline Serrapinus sp. & & & & & 7 & 7 & 14 & 0,66 \\
\hline Serrasalmus rhombeus & & & & & & 1 & 1 & 0,05 \\
\hline Steindachnerina sp. & & & & & & 1 & 1 & 0,05 \\
\hline Triportheus angulatus & & & & & & 2 & 2 & 0,09 \\
\hline Tyttocharax sp. & & & & 3 & 11 & & 14 & 0,66 \\
\hline Crenuchidae & & & & & & & & \\
\hline Characidium etheostoma & & & 3 & & 9 & 3 & 15 & 0,71 \\
\hline Characidium sp. & & & 2 & & & & 2 & 0,09 \\
\hline Geryichthys sterbai. & & & & & 1 & & 1 & 0,05 \\
\hline Melanocharacidium $s p$. & & & 5 & & 1 & 2 & 8 & 0,38 \\
\hline $\begin{array}{l}\text { Erythrinidae } \\
\text { Hoplias malabaricus }\end{array}$ & & & & & 1 & 2 & 3 & 0,14 \\
\hline Prochilodontidae & & & & & & & & \\
\hline Prochilodus nigricans & & & & 1 & & & 1 & 0,05 \\
\hline Curimatidae & & & & & & & & \\
\hline Cyphocharax sp. & & & & & 2 & & 2 & 0,09 \\
\hline Steindachnerina guentheri & & & & & 18 & 4 & 22 & 1,04 \\
\hline Steindachnerina hypostoma & & & & 2 & & 6 & 8 & 0,38 \\
\hline Anostomidae & & & & & & & & \\
\hline $\begin{array}{l}\text { Leporinus sp. } \\
\text { Lebiasinidae }\end{array}$ & & & & & & 5 & 5 & 0,24 \\
\hline $\begin{array}{l}\text { Lebiasinidae } \\
\text { Pyrrhulina sp. }\end{array}$ & & & & & 42 & & 42 & 1,99 \\
\hline Hemiodontidae & & & & & & & & \\
\hline Hemiodus $s p$. & & & & & & 1 & 1 & 0,05 \\
\hline \multicolumn{9}{|l|}{ GYMNOTIFORMES } \\
\hline Apteronotus anas & & & & & & 1 & 1 & 0,05 \\
\hline Rhamphichthyidae & & & & & & & & \\
\hline Rhamphichthys rostratum & & & & & & 1 & 1 & 0,05 \\
\hline \multirow{2}{*}{\multicolumn{9}{|c|}{$\begin{array}{l}\text { SILURIFORMES } \\
\text { Aspredinidae }\end{array}$}} \\
\hline & & & & & & & & \\
\hline \multirow{2}{*}{\multicolumn{9}{|c|}{ Heptapteridae }} \\
\hline & & & & & & & & \\
\hline Imparfinis sp. & & & & 9 & 8 & & 17 & 0,80 \\
\hline Pimelodella gracillis & & & & & & 1 & 1 & 0,05 \\
\hline Heptapterus sp. & & & & & 2 & & 2 & 0,09 \\
\hline Rhamdia quelen & 3 & 1 & & & & & 4 & 0,19 \\
\hline \multicolumn{9}{|l|}{ Loricariidae } \\
\hline Chaetostoma lineopuntatum & 14 & 1 & 4 & & & & 19 & 0,90 \\
\hline Ancistrus sp. & 8 & 1 & & & 1 & & 10 & 0,47 \\
\hline Rineloricaria $s p$. & & & & 1 & & & 1 & 0,05 \\
\hline Crossoloricaria sp. & & & & 8 & & & 8 & 0,38 \\
\hline Loricaria $s p$. & & & & 1 & & 16 & 17 & 0,80 \\
\hline Liposarcus disjuntivus & & & & 1 & & & 1 & 0,05 \\
\hline Aphanotorulus unicolor & & & & & & 10 & 10 & 0,47 \\
\hline Hypostomus sp. & 2 & & & & & & 2 & 0,09 \\
\hline Pimelodidae & & & & & & & & \\
\hline Pimelodinae (alevino) & & & & 1 & & & 1 & 0,05 \\
\hline Pimelodina sp. & & & & & & 1 & 1 & 0,05 \\
\hline Hypophthalmus edentatus & & & & & & 1 & 1 & 0,05 \\
\hline Pimelodus pictus & & & & & & 1 & 1 & 0,05 \\
\hline Trichomycteridae & & & & & & & & \\
\hline Pseudostegophilus nemurus. & & & & & & 3 & 3 & 0,14 \\
\hline Vandellia cirrhosa & & & & 1 & & 4 & 5 & 0,24 \\
\hline BELONIFORMES & & & & & & & & \\
\hline Belonidae & & & & & & & & \\
\hline Potamorrhaphis sp. & & & & & & 2 & 2 & 0,09 \\
\hline Pseudotylosurus angusticeps & & & & & & 1 & 1 & 0,05 \\
\hline & & & & & & & Cont & inúa... \\
\hline
\end{tabular}


Apéndice 4. Continuación.

\begin{tabular}{|c|c|c|c|c|c|c|c|c|}
\hline Orden / Familia / Especie & TARA & CAIN & SABA & PONG & COPU & YURI & Total & $\%$ \\
\hline \multicolumn{9}{|l|}{ Poecilidae } \\
\hline Poecilia reticulata & & & & & 1 & & 1 & 0,05 \\
\hline \multicolumn{9}{|l|}{ CYPRINODONTIFORMES } \\
\hline \multicolumn{9}{|l|}{ Rivulidae } \\
\hline Rivulus & 26 & 29 & 2 & & & & 57 & 2,70 \\
\hline \multicolumn{9}{|l|}{ SYNBRANCHIFORMES } \\
\hline \multicolumn{9}{|l|}{ Synbranchidae } \\
\hline Synbranchus marmoratus & & 1 & & & & & 1 & 0,05 \\
\hline \multicolumn{9}{|l|}{ PERCIFORMES } \\
\hline \multicolumn{9}{|l|}{ Cichlidae } \\
\hline Aequidens tetramerus & & & & & 8 & & 8 & 0,38 \\
\hline Apistogramma sp. 1 & & & & & 8 & & 8 & 0,38 \\
\hline Apistogramma sp. 2 & & & & & 9 & & 9 & 0,43 \\
\hline Apistogrammoides sp. & & & & & 4 & & 4 & 0,19 \\
\hline Bujurquina huallagae & 5 & & & 59 & 138 & 9 & 211 & 9,99 \\
\hline Bujurquina sp. & 1 & & & & & & 1 & 0,05 \\
\hline Cichla monoculus & & & & & & 1 & 1 & 0,05 \\
\hline Cichlasoma amazonarum & & & & 1 & & 1 & 2 & 0,09 \\
\hline Crenicichla sp. & & & & 1 & 1 & & 2 & 0,09 \\
\hline \multicolumn{9}{|l|}{ Sciaenidae } \\
\hline Plagioscion squamossisimus & & & & & & & 1 & 0,05 \\
\hline 95 & 377 & 239 & 21 & 332 & 533 & 612 & 2113 & 100 \\
\hline
\end{tabular}


童| 